\title{
Minimum time to use the fluid model in dc electrical discharges
}

\begin{abstract}
B. Ardjani and B. Liani
Laboratory of Theoretical Physics, Science Faculty, Tlemcen University, P. O. Box 119, 13000 Tlemcen, Algeria.

Accepted 13 August, 2012

In the study of thermal equilibrium of the electrons, it is very necessary to use the fluid model method. In this work, effect of the gas density, the electrons initial energy and the electric field on the electron thermal equilibrium is studied. It is found that the steady state can be achieved by increasing the gas density or the inter-electrode distance. If the gas density or the inter-electrode distance are not sufficient, steady state can be achieved by increasing the electric field. The initial energy of the electrons has great effect on the steady state if the electric field is weak and the difference between the initial energy and the electrons mean energy is great, however this problem can be solved by extending the time. It is necessary to make a best combination between the gas density, inter-electrode distance and the electric field to make a modeling by the fluid model method in the best cases of the steady state. A simple formula is given here to find the minimum time required to use the fluid model for $E / N \geq$ $500 \mathrm{Td}$.
\end{abstract}

Key words: Electrons thermal equilibrium, transport properties, fluid model.

\section{INTRODUCTION}

Fluid model is often used in the simulation of the dc electrical discharges, for example streamer discharges (Chao Li et al., 2007; Kulikovsky, 1995), dc glow discharges (Bogaerts, 1999; Baguer et al., 2003; Farouk et al., 2006).

The fluid model method cannot be used only if the steady state of the transport parameters is achieved. The equilibrium depends on different parameters such as the electric field strength, electron initial energy and the gas density, the effect of these parameters on the steady state of the transport parameters is well discussed in this work.

Also if the time is not sufficient the steady state is not achieved and the fluid model method cannot be used. A simple formula to find minimum time to use the fluid model method at high electric field is given here.

\section{METHOD OF SIMULATION}

The Monte Carlo collision method (MCC) described by Ardjani and Liani (2009) is used in this work. In the MCC method, firstly, the free flight time between two successive collisions is calculated by generating random numbers (between 0 and 1). The free flight time is used in the calculation of the equations of motion; therefore the electron energy changes under the acting of the electric field. The collision process (elastic, excitation, ionization, attachment) is selected by generating a random number between 0 and 1 and according to the electron energy, the electron energy decreases according to the collision nature. Finally several parameters are registered to be used in the calculation of the transport parameters as is well clarified by Ardjani and Liani (2009).

*Corresponding author. E-mail: benamarxxm@yahoo.fr.

PACS: 52.25.Fi, 52.65.Kj. 
Table 1. Considered reactions of $\mathrm{N}_{2}$ with his levels and energy range (Yousfi et al., 1988).

\begin{tabular}{lcc}
\hline Reactions & Levels & Energy range $(\mathrm{eV})$ \\
\hline Momentum transfer & & $0-1000$ \\
Molecule rotation & & $0.03-3.6$ \\
Molecule vibration & $\mathrm{v}=1$ & $0.3-50$ \\
Molecule vibration & $\mathrm{v}=1$ & $1.65-3.6$ \\
Molecule vibration & $\mathrm{v}=2$ & $1.8-3.5$ \\
Molecule vibration & $\mathrm{v}=3$ & $2-3.3$ \\
Molecule vibration & $\mathrm{v}=4$ & $2.1-3.2$ \\
Molecule vibration & $\mathrm{v}=5$ & $2.2-3.3$ \\
Molecule vibration & $\mathrm{v}=6$ & $2.3-3.1$ \\
Molecule vibration & $\mathrm{v}=7$ & $2.4-3.4$ \\
Molecule vibration & $\mathrm{v}=8$ & $2.6-3.4$ \\
Molecule vibrational excitation & & $7-70$ \\
Molecule vibrational excitation & & $7.3-70$ \\
Molecule excitation & & $8-70$ \\
Molecule excitation & & $8-100$ \\
Molecule excitation & & $8.1-70$ \\
Molecule excitation & & $9-70$ \\
Molecule excitation & & $9-150$ \\
Molecule excitation & & $9-1000$ \\
Molecule excitation & & $9.1-50$ \\
Molecule excitation & & $11.5-100$ \\
Molecule excitation & & $13.92-50$ \\
Molecule excitation & & $14-1000$ \\
Molecule excitation & & $16-1500$ \\
Total ionization & &
\end{tabular}

\section{RESULTS AND DISCUSSION}

The electrons are directed parallel to the $z$ axis initially, but after the first collision the electrons are scattered randomly in 3-dimensional space. The gas used is the nitrogen, the reactions considered are: the momentum transfer, 1 molecule rotation, 9 molecule vibrations, 2 vibrational excitations, 11 excitations, total ionization. These are listed in the Table 1.

For comparison of the results with available literatures, Figure 1 shows the electrons energy and drift velocity calculated by this code with the calculations of Phelps and Pitchford (1985). Good agreement is shown specially for the drift velocity.

\section{The gas density effect}

Figure 2 shows the temporal mean energy of the electrons in the case of the nitrogen with different gas densities $\left(T=300^{\circ} \mathrm{K}\right.$ and $\mathrm{P}=0.1,0.2,0.4,2$ Torr $)$ at $\mathrm{E} / \mathrm{N}$ $=150 \mathrm{Td}$. At fixed time (100 ns) or rather at fixed interelectrode distance the choice of the gas density has direct effect on the steady state and on the values of the transport parameters. Figure 2 shows that for 0.1 and 0.2
Torr the steady state is not achieved, but it begins approximately from 0.4 Torr, the best case of the steady state is for 2 Torr. The number of collisions increases proportionally to the increase of the gas density. The steady state is obtained when the number of collisions is sufficient to make good distribution of the energy between the electrons. As a result, at fixed inter-electrode distance the steady state is obtained by increasing the gas density, or at fixed gas density the steady state is obtained by increasing the inter-electrode distance.

\section{The electrons initial energy effect}

Figure 3 shows the temporal mean energy of the electrons in the case of the nitrogen at $\mathrm{E} / \mathrm{N}=20,100$, $150 \mathrm{Td}$ with different initial energies of the electrons.

For $\mathrm{E} / \mathrm{N}=20 \mathrm{Td}$ the steady state is achieved for $\varepsilon_{0}=1$ $\mathrm{eV}$, but for $\varepsilon_{0}=4 \mathrm{eV}$ the mean energy is far to the steady state. The initial energy has great effect on the steady state when the difference between the initial energy and the mean energy is great. Generally the steady state is not influenced by the initial energy of the electrons for relatively high electric field (in this case approximately for $E / N \geq 100 \mathrm{Td}$ ), but if the initial energy increases the 


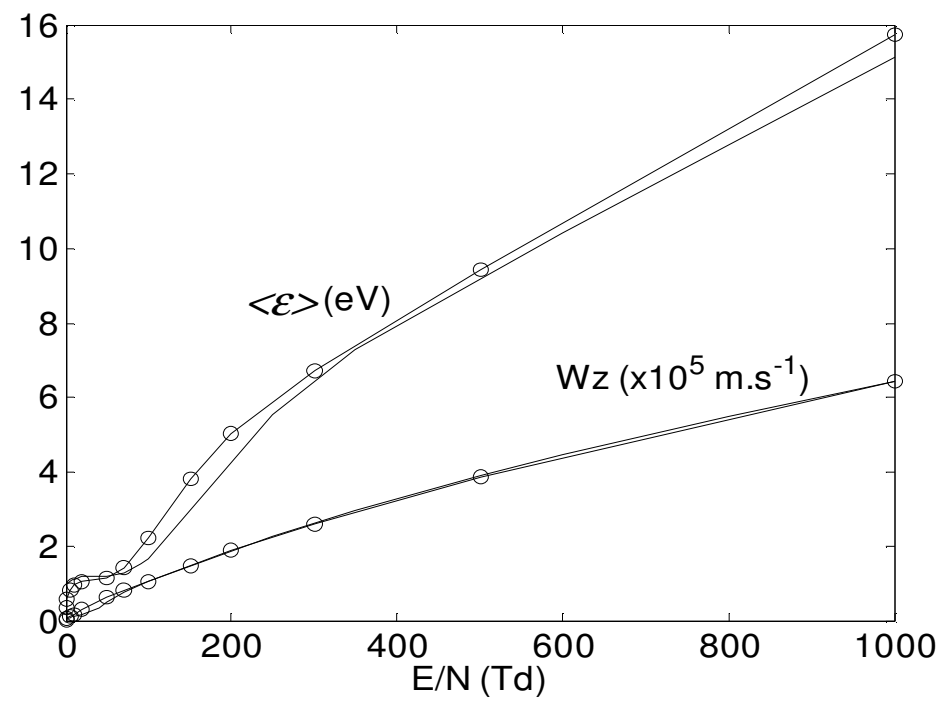

Figure 1. Mean energy and drift velocity as function of $E / N(T d)$ for $\mathrm{N}_{2}$ [solid line: this work; solid line with circles: Phelps and Pitchford (1985)].

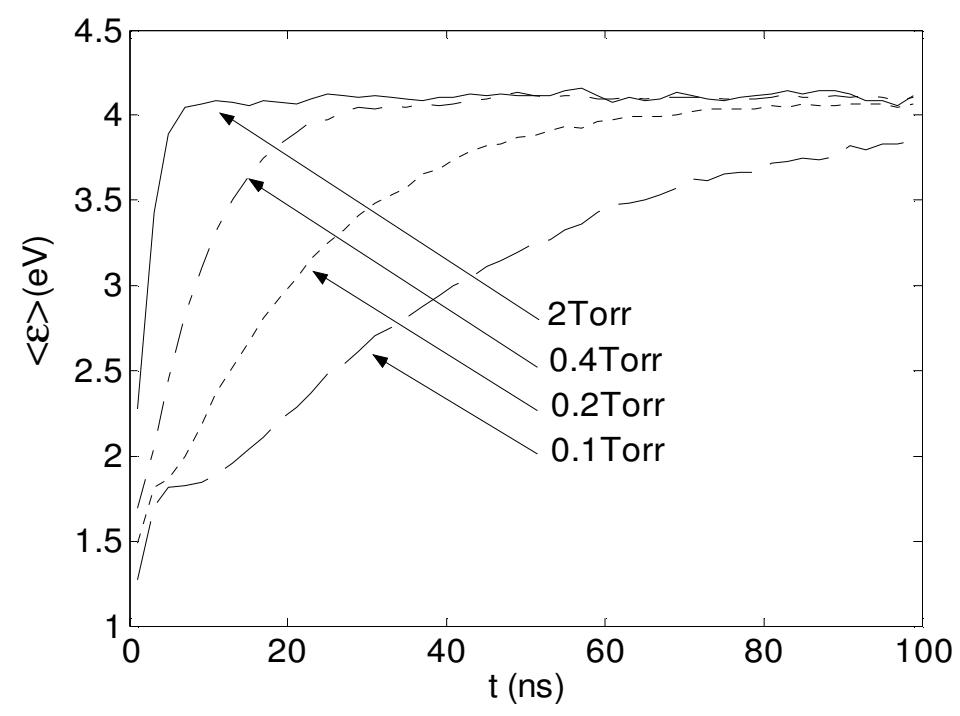

Figure 2. Temporal mean energy of the electrons in the case of the nitrogen with different gas densities $\left(T=300^{\circ} \mathrm{K}\right.$ and $\mathrm{P}=0.1,0.2,0.4,2$ Torr) at $\mathrm{E} / \mathrm{N}=150 \mathrm{Td}$.

steady state will be more delayed. Also the perfect steady state (which means that the stationary mean energy is from the beginning to the end) is obtained when the initial energy equals the mean energy and $\mathrm{E} / \mathrm{N} \leq 100 \mathrm{Td}$ (for example, the cases of $\mathrm{E} / \mathrm{N}=20 \mathrm{Td}, \varepsilon_{0}=1 \mathrm{eV}$ and $\mathrm{E} / \mathrm{N}=$ $\left.100 \mathrm{Td}, \varepsilon_{0}=2.5 \mathrm{eV}\right)$. The perfect steady state allows the perfect use of the fluid model method; also the first region of non equilibrium must be greatly reduced to make the best use of the fluid model method. As a result it is necessary to best combine the gas density, the inter- electrode distance and the electric field to make a modeling of the electrical discharges by the fluid model method in the best cases of the steady state.

Figure 4 shows the temporal mean energy of electrons in the case of nitrogen at $\mathrm{E} / \mathrm{N}=20 \mathrm{Td}$ with $\varepsilon_{0}=4 \mathrm{eV}$, and with the time extended to 600 ns. Figure 4 shows that if the mean energy is not in the steady state since the initial energy is high the equilibrium can be achieved by extending the time or by increasing the gas density.

Figure 5 shows the electron energy distribution function 


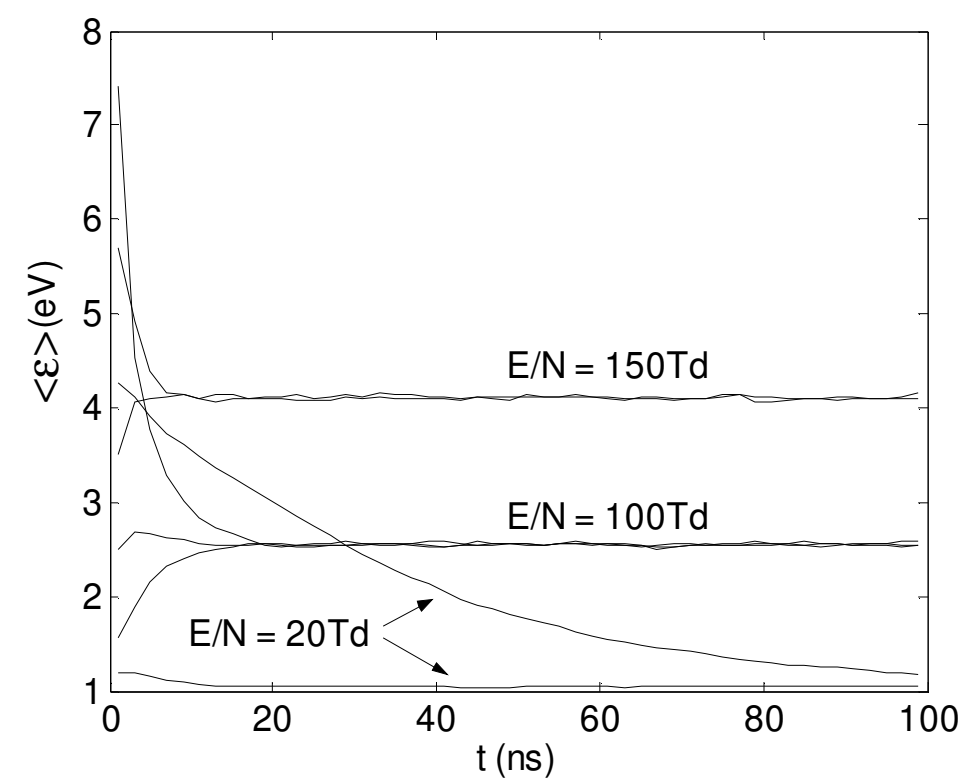

Figure 3. Temporal mean energy of electrons in the case of the nitrogen at $\mathrm{E} / \mathrm{N}=20 \mathrm{Td}\left(\varepsilon_{0}=1,4 \mathrm{eV}\right), \mathrm{E} / \mathrm{N}=100 \mathrm{Td}\left(\varepsilon_{0}=1,2.5,7 \mathrm{eV}\right)$, $\mathrm{EN}=150 \mathrm{Td}\left(\varepsilon_{0}=2.5,4 \mathrm{eV}\right)(\mathrm{P}=2 \mathrm{Torr})$.

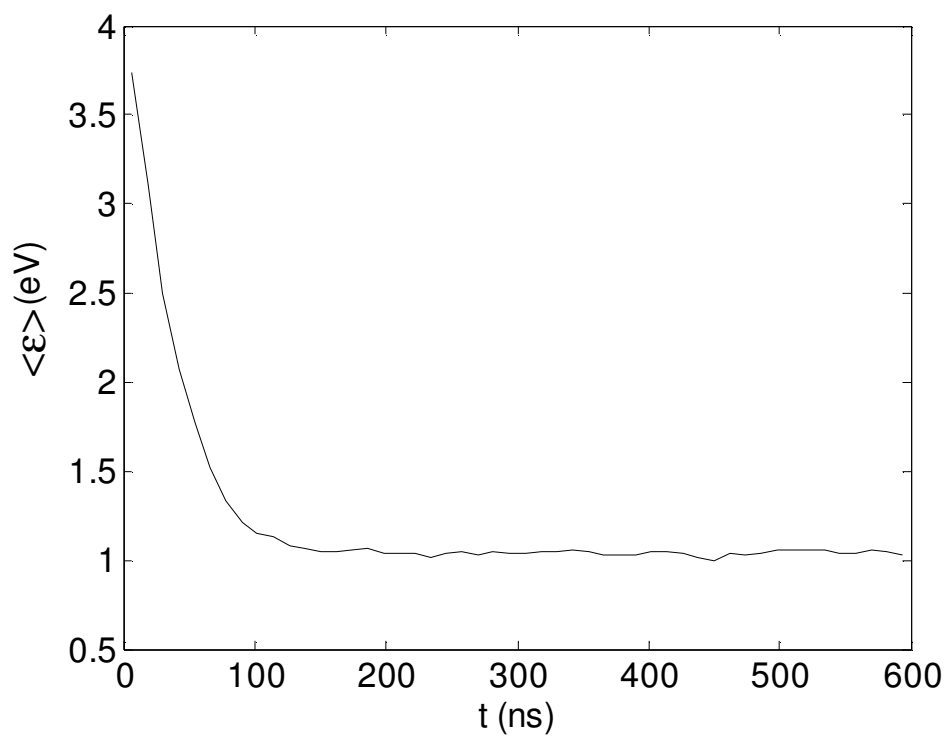

Figure 4. Temporal mean energy of electrons in the case of the nitrogen at $\mathrm{E} / \mathrm{N}=20 \mathrm{Td}$ with $\varepsilon_{0}=4 \mathrm{eV}$, and with the time extended to $600 \mathrm{~ns}(\mathrm{P}=$ 2 Torr).

(EEDF) in the case of the nitrogen at $\mathrm{E} / \mathrm{N}=100 \mathrm{Td}$ with different initial energies of the electrons. In the case of the nitrogen, the EEDF is Maxwellian for $\mathrm{E} / \mathrm{N} \geq 500 \mathrm{Td}$ (Tas et al., 1997). Figure 5 shows that when the steady state is achieved and the electric field is relatively high $(E / N \geq 100 \mathrm{Td})$, and if the initial energy of the electrons changes the EEDF has a negligible change.
Figure 6 shows the electron energy distribution function (EEDF) in the case of nitrogen at $\mathrm{E} / \mathrm{N}=20 \mathrm{Td}$ with different initial energies of the electrons. Figure 6 shows that when the steady state of the mean energy changes by the change of the initial energy, the EEDF changes also, but if the time is extended (to $600 \mathrm{~ns}$ in this case) the EEDF goes more and more to the steady state EEDF. 


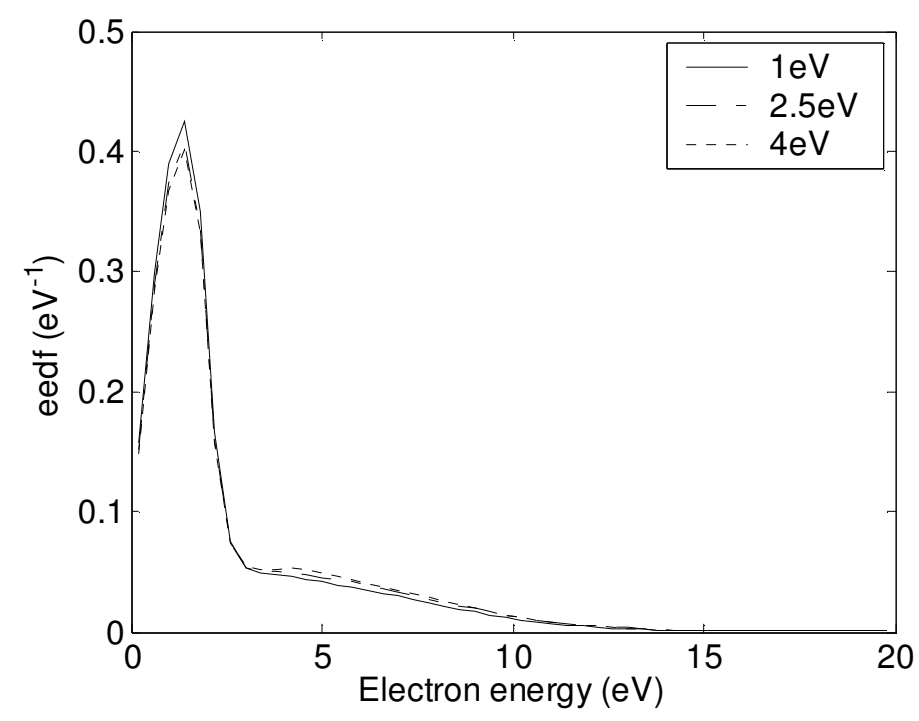

Figure 5. EEDF in the case of nitrogen at $\mathrm{E} / \mathrm{N}=100 \mathrm{Td}$ with $\varepsilon_{0}=1$, $2.5,4 \mathrm{eV}$.

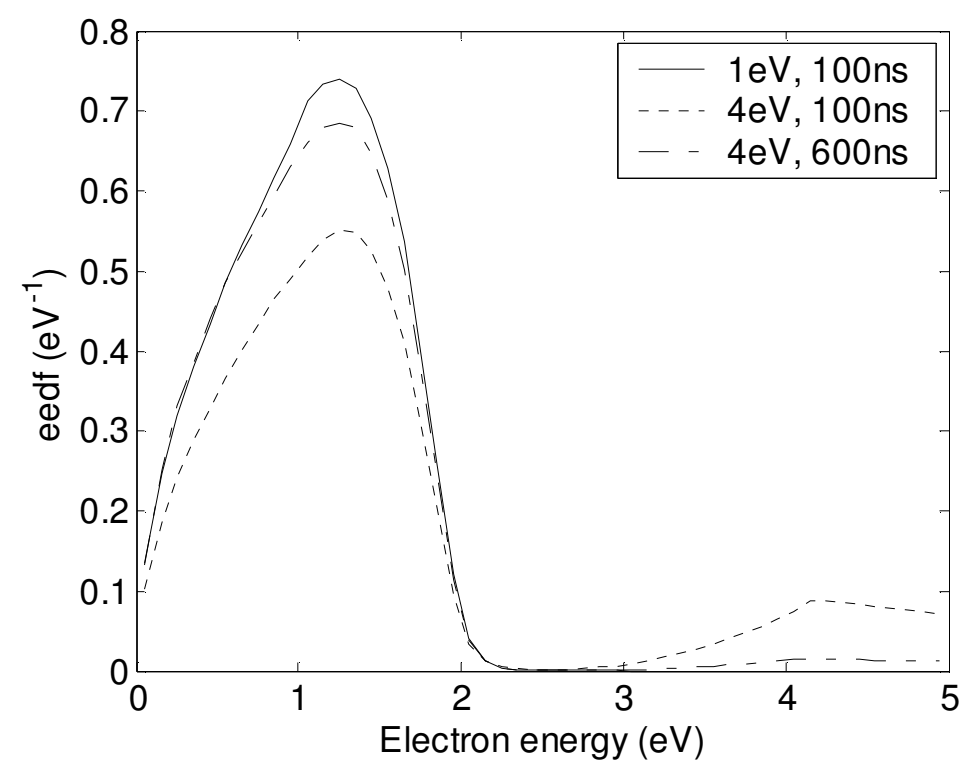

Figure 6. EEDF in the case of nitrogen at $E / N=20 \mathrm{Td}$ with $\varepsilon_{0}=1,4$ $\mathrm{eV}$ for $\mathrm{t}=100$ and $600 \mathrm{~ns}$, respectively.

\section{The electric field effect}

Figure 7 shows the temporal mean energy of the electrons in the case of nitrogen and argon at different values of the electric field, $\mathrm{Pg}=2$ Torr.

Figure 7 show that the electric field has a role to achieve the steady state. In the case of $\mathrm{N}_{2}$ when $\mathrm{E} / \mathrm{N}=$ $150 \mathrm{Td}$ the mean energy is not in the equilibrium, but for $\mathrm{E} / \mathrm{N}=300 \mathrm{Td}$ the equilibrium is achieved and the best case of the steady state begins from $E / N=500 \mathrm{Td}$.
Therefore if the gas density or inter-electrodes distance are not sufficient the equilibrium can be achieved by increasing the electric field.

\section{Required time to achieve the electron thermal equilibrium}

From Figure 7, for $\mathrm{N}_{2}$, approximately the same behavior of the mean energy is obtained if $\mathrm{E} / \mathrm{N} \geq 500 \mathrm{Td}$. 
(a)

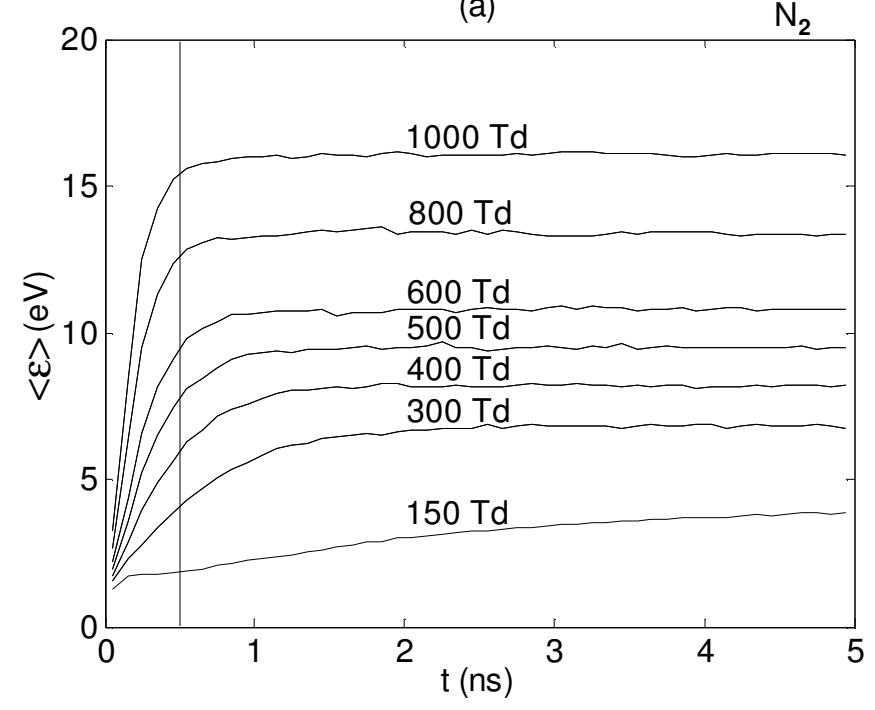

(b)

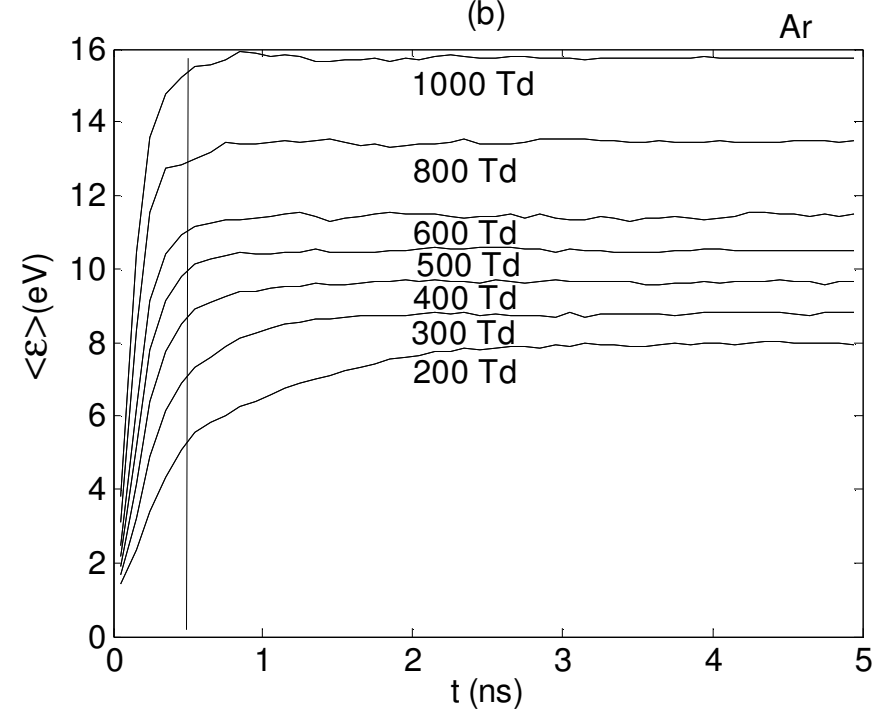

Figure 7. Temporal mean energy of the electrons at different values of $E / N, P=2$ Torr: (a) nitrogen, (b) argon.

Therefore one can say that the steady state is independent of the electric field if $E / N \geq 500 \mathrm{Td}$, as it depends only on the gas density. For the argon gas the same behavior of the mean energy is obtained if $E / N \geq$ $400 \mathrm{Td}$. Also the time to achieve the steady state is inversely proportional to the gas density; therefore the time to achieve the steady state when $\mathrm{E} / \mathrm{N} \geq 500 \mathrm{Td}$ can be deduced using the following relation:

$$
t_{e q}=C_{e t} / N_{g}
$$

Where $t_{e q}$ is the time required to achieve the steady state, $C_{e t}=3.14 \times 10^{14}$ is the equilibrium time coefficient and $N_{g}$ is the gas density.
Also the equilibrium time can be expressed as a function of the gas pressure at ambient temperature $\left(300^{\circ} \mathrm{K}\right)$ as follows:

$t_{e q}=C_{e t p} / P_{g}$

Where $C_{e t p}=1 \times 10^{-8}$ is the pressure coefficient of the equilibrium time.

The values of $C_{e t}, C_{e t p}$ given here are available for the nitrogen and the argon gas, but they can be changed slightly for other gases. The Equations (1) and (2) are available for $E / N \geq 500 \mathrm{Td}$ in the case of nitrogen and $\mathrm{E} / \mathrm{N} \geq 400 \mathrm{Td}$ in the case of argon; though the minimum 


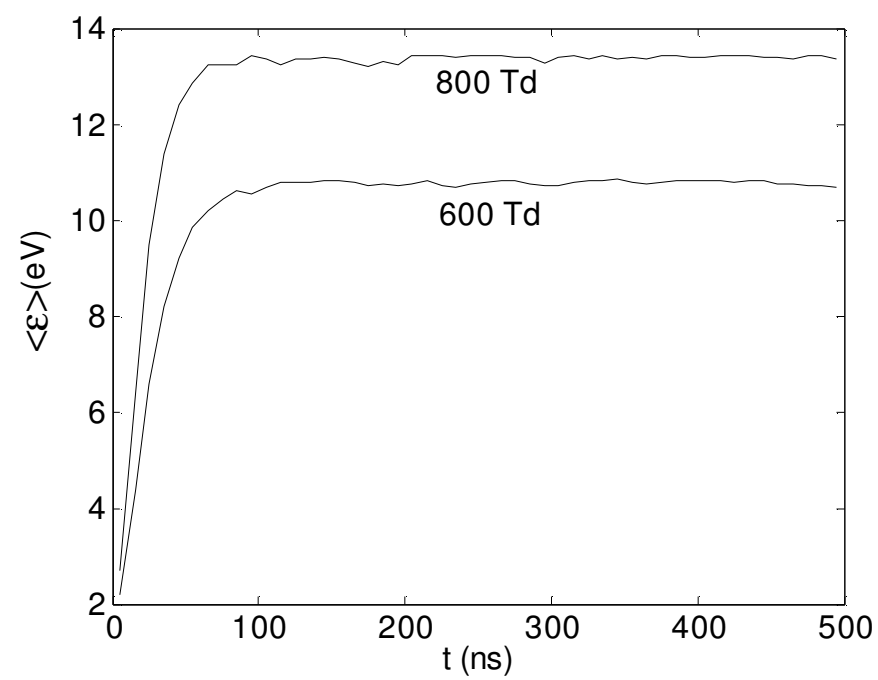

Figure 8. Temporal mean energy of the electrons in the case of nitrogen at $\mathrm{E} / \mathrm{N}=600$ and $800 \mathrm{Td}$ for $\mathrm{Pg}=0.02$ Torr.

$\mathrm{E} / \mathrm{N}$ can be changed for other gases, $\mathrm{E} / \mathrm{N} \geq 500 \mathrm{Td}$ is sufficient.

The equilibrium time given in Equations (1) and (2) is chosen to give best cases of the steady state, for modelling by the fluid model. This time is the minimum time to be used in this type of simulation.

For $\mathrm{E} / \mathrm{N} \leq 500 \mathrm{Td}$ the time of simulation depends on the electric field; when $\mathrm{E} / \mathrm{N}$ decreases the time of simulation must be increased. This makes it difficult to find a relation that gives the equilibrium time because the equilibrium time is not inversely proportional to the electric field.

Figure 8 shows the temporal mean energy of the electrons in the case of nitrogen at $\mathrm{E} / \mathrm{N}=600$ and 800 $\mathrm{Td}$, for $\mathrm{Pg}=0.02$ Torr. The relations (1) and (2) are tested by changing the gas pressure, for $P_{g}=0.02$ Torr; the equilibrium time required is $500 \mathrm{~ns}$. In the case of nitrogen at $\mathrm{E} / \mathrm{N}=600,800 \mathrm{Td}$ and $P_{g}=0.02$ Torr the same behavior of the mean energy is obtained compared to the case of $P_{g}=2$ Torr; it changes only the time of simulation.

\section{Conclusion}

In this work, effect of gas density, electric field and initial energy of the electrons on the electron thermal equilibrium is studied. It thus reveals that:

1) The steady state can be achieved by increasing the gas density or inter-electrode distance.

2) If the gas density or inter-electrode distance is not sufficient, the steady state can be achieved by increasing the electric field.

3) The initial energy of the electrons has great effect on the steady state if the electric field is low and the difference between the initial energy and the electrons mean energy is great, but this problem can be solved by extending the time.

4) It is necessary to best combine the gas density, interelectrode distance and electric field to make a modeling of the electrical discharges by the fluid model method in the best cases of the steady state.

5) Simple formulas of the minimum time needed to achieve the electron thermal equilibrium are provided.

\section{REFERENCES}

Ardjani B, Liani B (2009). The validity of using the transport parameters of the Townsend discharge in the simulation of the of and DBD discharges by the fluid model. FIZIKA A (Zagreb) 18(3):107-120.

Baguer N, Bogaerts A, Gijbels R (2003). Hollow cathode glow discharge in $\mathrm{He}$ : Monte Carlo-Fluid model combined with a transport model for the metastable atoms. J. Appl. Phys. 93(1).

Bogaerts A (1999). Comprehensive modelling network for dc glow discharges in argon. Plasma Sources Sci. Technol. 8:210-229.

Chao Li, Brok WJM, Ebert U, van der Mullen JJAM (2007). Deviations from the local field approximation in negative streamer heads. J. Appl. Phys. 101:123305.

Farouk T, Farouk B, Staack D, Gutsol A, Fridman A (2006). Simulation of dc atmospheric pressure argon micro glow-discharge. Plasma Sour. Sci. Technol. 15:676-688.

Kulikovsky AA (1995). Two-dimensional simulation of the positive streamer in N2 between I parallel-plate electrodes. J. Phys. D. Appl. Phys. 28:2483-2493.

Phelps AV, Pitchford LC (1985). Anisotropic scattering of electrons by $\mathrm{Nz}$ and its effect on electron transport. Phys. Rev. A 31(5):2932.

Tas MA, van Veldhuizen EM, Rutgers WR (1997). Plasma excitation processes in flue gas simulated with Monte Carlo electron dynamics. J. Phys. D: Appl. Phys. 30:1636-1645.

Yousfi M, Azzi N, Gallimberti I Stangherlin S (1988). Electron-molecule collision cross sections and electron swarm parameters in some atmospheric gases. Collection data $\mathrm{n}^{\circ}{ }^{1}$, Internal report. Toulouse Padova. 